\title{
Experimental study to identify the effect of type of coupling on unbalance using frequency spectrum analysis
}

\author{
Shamim Pathan ${ }^{1}$, Pallavi Khaire ${ }^{2}$ \\ ${ }^{I}$ (Mechanical Engineering Department, Fr.C.Rodrigues Institute of Technology, University of Mumbai, India) \\ ${ }^{2}$ (Mechanical Engineering Department, Fr.C.Rodrigues Institute of Technology, University of Mumbai, India)
}

\begin{abstract}
Any defect in machine will affect vibration behavior and nature of this effect is different for different faults. Understanding and practicing the fundamentals of rotating shaft parameters is the first step in reducing unnecessary vibration, reducing maintenance costs and increasing machine uptime. Hence condition monitoring based on vibration measurements can be used to identify those defects qualitatively. Vibration signals give early indication of mechanical failures such as misalignment, unbalance and bent shaft etc. The present study deals with experimental investigation of unbalance with the help of FFT analyzer and its unique vibration spectrum for different types of couplings such as jaw coupling, flexible flange coupling and rigid coupling. Understanding and practicing the fundamentals of rotating shaft parameters is the first step in reducing unnecessary vibration, reducing maintenance costs and increasing machine uptime. Many researchers have reported unbalance related faults show dominant peak at $1 \times$. For experimental identification of unbalance an experimental setup is constructed and frequency spectrum is acquired for jaw coupling, flexible flange coupling and rigid flange coupling. These acquired spectrums are compared with available literature for confirmation of experiment results. The experimental results are in close agreement with results available in literature.
\end{abstract}

Keywords: Coupling, FFT analyzer, Unbalance.

\section{Introduction}

Rotor systems have been widely used in mechanical engineering. The dynamics of rotor systems have been studied for over a century. With the high speed demand of today's machinery, it becomes more important than ever. A rotating machine has one or more machine elements that rotate with a shaft, such as rolling-element bearings, impellers, and other rotors. In a perfectly balanced machine, all rotors rotate true on their centerline and all forces are equal. In industrial machinery, it is a common feature that an imbalance of forces to occur. In addition to imbalance generated by a rotating element, vibration may be caused by instability, therefore it can be concluded that the main causes of mechanical vibration are unbalance, misalignment, looseness, distortion, defective bearings, gearing defect, coupling inaccuracies, rotor shaft misalignment, bent rotor shaft and so on. The vibration caused by unbalance may destroy critical parts such as bearings, seals, gears and couplings. In real system, faults are inevitable due to errors in manufacturing provision of tolerances on mating parts, errors while assembling different parts of the system, faults may develop in the system due to operating conditions such as heat generation, looseness and wear etc. Therefore identification of above fault in machine is very important to avoid catastrophic failure. The defect in a rotor system will affect vibration behavior and nature of this effect is different for different types of faults .Therefore it can be said that the vibration signals are early indication of mechanical failures. The identification of faults at early stage is becoming an important aspect of industrial strategy to avoid breakdown of machinery. In this regard condition based maintenance based on vibration analysis can be effectively used to qualitatively identify faults.

Many researchers have studied techniques of faults diagnosis related to unbalance. M.Xu and Marangoni[1][2] have developed a theoretical model of a complete motor-flexible-coupling-rotor system using the component mode synthesis. General system of equation of motion derived for a system under misalignment and unbalance condition. The derived equations indicate that forcing frequency due to shaft misalignment are even multiple frequencies of the motor rotational speed. They also validated their theoretical results with experimental study. A.Askarian,S.M.R. Hashemi[3] studied effect of axial force, unbalance and coupling misalignment on vibration of rotor gas turbine. It is reported that for unbalance highest amplitude is at $1 \mathrm{X}$ of the shaft speed and for misalignment highest amplitude is at $2 \mathrm{X}$ of the shaft speed. V.Hariran ,P.S.S. Srinivasan[4] studied the effect of unbalance on a rotor system with flexible flange coupling. The experimental and numerical spectra were obtained for unbalance under different unbalance forces. They concluded that both experimental and numerical results show the dominant peak at $1 \times$ and experimental results are in good agreement with numerical results. It is revealed from the literature study that it is important to detect the fault at earlier stage, so that the machine life can be enhanced with less cost. It is clearly proven that misalignment produces high vibration level in bearings and couplings. Therefore it is proposed to investigate experimentally the effect of 
unbalance on frequency spectrum for three different couplings viz. jaw coupling, flexible flange coupling and rigid flange coupling and validate the typical spectrum reported in literature.

\section{Experimental setup}

The picrtorial view of experimental setup is shown in fig. 1. The experimental setup consist of a 0.25 HP d.c motor with extended shaft, a jaw coupling, single disk rotor and three identical self aligned ball bearing. The shaft is supported by two bearing and has a length of $430 \mathrm{~mm}$ with a bearing span of $300 \mathrm{~mm}$. The diameter of shaft is $15.875 \mathrm{~mm}$. A disk $80 \mathrm{~mm}$ diameter and $10 \mathrm{~mm}$ thick is made of steel mounted on shaft. The two holes of diameter $10 \mathrm{~mm}$ are drilled diametrically opposite to each other to attach desire amount of unbalance mass.

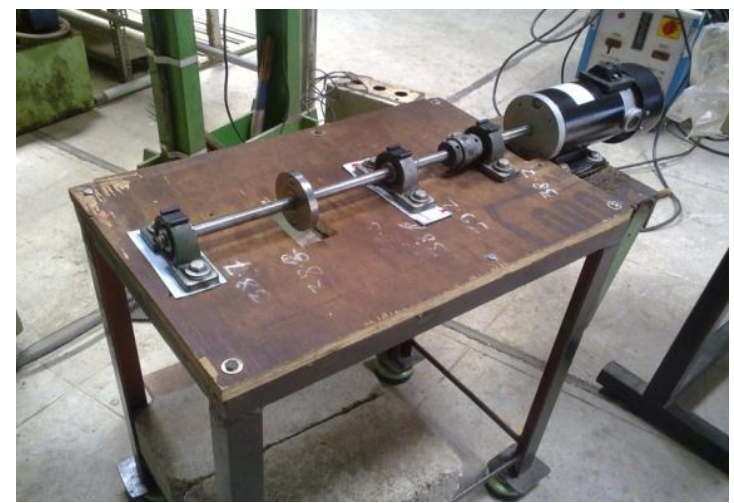

Fig.1:The experimental setup

Pedestal bearings are used in setup for supporing motor shaft and two bearing are provided to support driven shaft and disc is mounted at the center of driven shaft. Initially, a jaw coupling is used to connect motor shaft and driven shaft. Later the jaw coupling is replaced by four pin type flexible flange coupling and then rigid coupling. The bearing which is near to the coupling on driven side is called as middle bearing. Bearing fitted at the end of driven shaft is called as end bearing. A disk which is mounted at center of shaft and mass of disc is $0.4 \mathrm{~kg}$. The purpose of mounting disk is to attach a mass for creating unbalance in experimental setup. The nut and bolt are used as unbalance mass which can be easily removed for balancing the system. A piezoelectric, Triaxial, shear type accelerometer (Type AC102-A, S1. No 66760) is used along with the Photon+ (Bruel and Kajer make) ultra portable dynamic signal analyzer. This is a multichannel data recorder. The data acquired by Photon+ data recorder is processed with FFT software and then the measured vibration data are collected at a computer terminal through RT-PHOTON+ interface. Vibrations are measured in frequency domain. Therefore output display in terms of frequency vs amplitude graph is selected in RT-PHOTON software. Vibration amplitude is measured in terms of displacement. For measuring 1600 spectral lines, frequency band of 0-60 Hz.The vibration sensor is mounted on top of bearings for acquiring signal. A speed regulator is used to vary the motor speed.

\section{Experimental procedure}

Initially, the setup is allowed run for a few minutes to allow all minor vibrations to settle. Before creating the unbalance, the shaft is checked for alignment. To do this, the dial gauge method is used to make perfect alignment. Also, the surface level is checked by using spirit level. Then, the accelerometer is fitted over the bearing housing and connected with the FFT analyzer. Next, the vibration data measured by using FFT analyzer and saved in a computer. A typical vibration spectrum is acquired on all three bearings for $900 \mathrm{rpm}(15 \mathrm{~Hz})$ to study the behavior of vibration frequency spectrum at balanced condition. To introduce unbalance in the system a mass of 30 gram is attached to disk at a location of $30 \mathrm{~mm}$ from the center. Two holes are drilled diametrically opposite on the disk at equidistance $30 \mathrm{~mm}$ from centre. The disk is fixed to shaft with a square key. The vibration sensor is placed on top of bearing blocks to acquire vibration spectrum for unbalanced condition radial direction Vibration spectrum is acquired for both balanced and unbalanced condition at rotor operating frequency 15Hz.After acquiring spectrum for jaw coupling, the coupling is changed and same procedure is followed for flexible flange coupling and rigid flange coupling.

\section{Results And Discussion}

The objective of this study is to investigate the effect of type of coupling and unbalance on frequency spectrum. The results of experimentation are discussed as follows. 


\section{A. Jaw coupling}

A typical vibration spectrum on middle bearing at a frequency of $15 \mathrm{~Hz}(900 \mathrm{rpm})$ for balanced and unbalanced case is given in fig. 2 and fig. 3 respectively. Fig. 2 shows peaks at $1 \times, 2 \times$ and $3 \times$.The highest amplitude is observed at $1 \times$ and its magnitude is $0.0041 \mathrm{~mm}$.Form fig. 3 it is observed that the unbalance effect shows dominant peak at $1 \times$ and there is increase in vibration level as compared to balanced condition. The amplitude of vibration for unbalanced condition is $0.023 \mathrm{~mm}$. The vibration spectrum is also acquired for other two bearings.

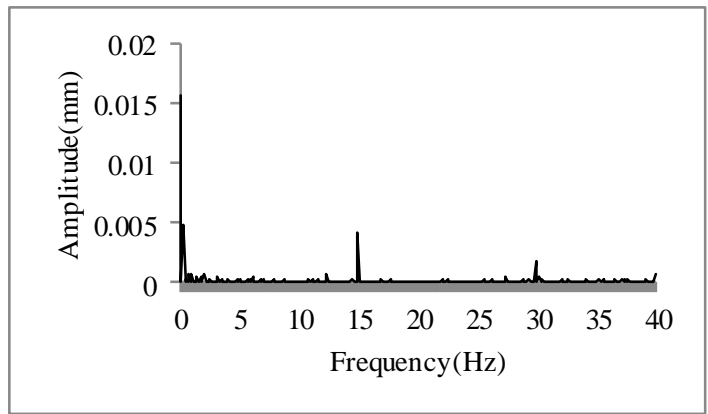

Fig.2: Frequency spectrum at $900 \mathrm{rmp}(15 \mathrm{~Hz})$ for

Fig.3: Frequency spectrum at $900(15 \mathrm{~Hz})$

balanced condition using jaw coupling

\section{B. Flexible flange coupling}

The frequency spectrum for four pin type flexible flange coupling is acquired on all three bearing at $15 \mathrm{~Hz}(900 \mathrm{rpm})$ for balanced condition and unbalanced condition. Frequency spectrum foe balanced condition is shown in fig.4. Fig.4 shows peak at $1 \times$ and the magnitude of amplitude is $0.012 \mathrm{~mm}$. Fig. 5 indicates the severity and unique characteristics of unbalance showing dominant peak at $1 \times$.The amplitude of vibration for unbalanced condition for flange coupling is $0.303 \mathrm{~mm}$.

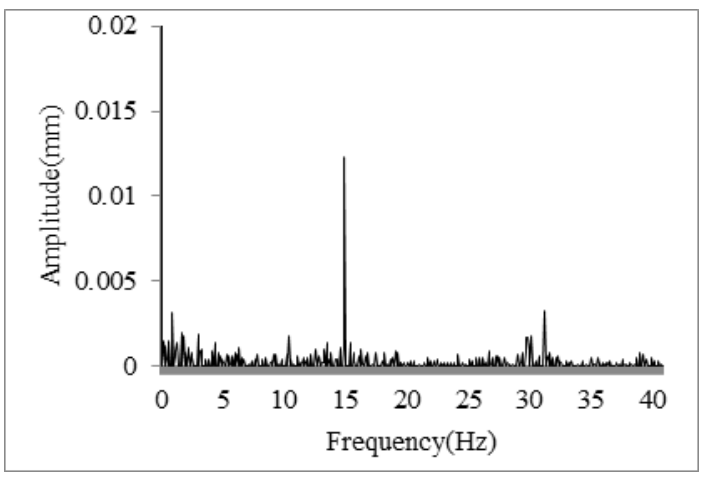

Fig.4 : Frequency spectrum at $900 \mathrm{rmp}(15 \mathrm{~Hz})$ for balanced at $900 \mathrm{rpm}(15 \mathrm{~Hz})$ for

condition using flexible flange coupling

flexibleflange coupling

\section{Rigid flange coupling}

For rigid flange coupling the maximum amplitude is seen at $1 \times$ for balanced and unbalanced conditoinon all bearings.Fig.6 and fig.7 shows frequency spectrum for balanced and unbalanced condition respectively for rigid coupling.

It is observed from above discussion that the highest vibration level is seen for rigid flange coupling for both balanced and unbalanced condition. All above couplings show the dominant peak on $1 \times$ for unbalanced condition and peaks at $1 \times, 2 \times$ and $3 \times$ for balanced condition. Using jaw coupling there is reduction in vibration level as compared to other two bearings. 


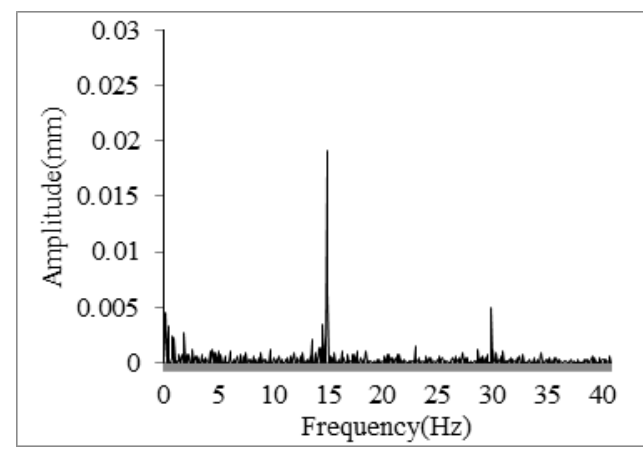

Fig.6:Frequency spectrum at $900 \mathrm{rpm}$ for balanced condition using rigid flange coupling

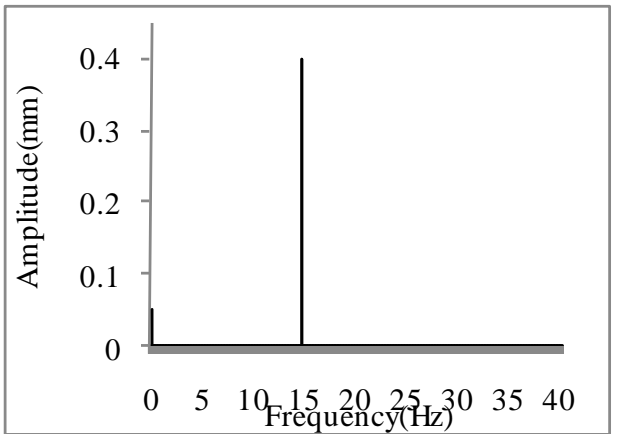

Fig.7: $\quad$ Frequency spectrum at $900 \mathrm{rpm}$ for unbalanced condition using rigid flange coupling

\section{Conclusion}

It is found from the experimental study that the unbalance shows dominant peak at $1 \times \mathrm{rpm}$. This unique signature is independant of type of coupling. Rigid coupling is more prone to vibrations as compared to other two couplings in unbalanced condition. It is also observed that using jaw coupling, the vibration level experienced is less. Hence it is concluded that the flexible flange coupling can resists the vibration due to unbalance in better way. These observations are in close agreement as reported in literature.

\section{References}

[1]. M.Xu ,R.D. Marangoni, Vibration Analysis of Motor-Flexible Coupling-Rotor System Subject to Misalignment and Unbalance, Part-I:Theoretical Model and Analysis, Journal of Sound and Vibration, (1994), pg.663-679.

[2]. M.Xu ,R.D. Marangoni, Vibration Analysis of Motor-Flexible Coupling-Rotor System Subject to Misalignment and Unbalance, Part-II: Experimental Validation, Journal of Sound and Vibration, (1994), pg.681-691.

[3]. AbdolrezaAskarian, S.M.R. Hashemi,Effect of axial force, unbalance and coupling misalignment on vibration of a rotor gas turbine, 14th international congress on sound vibration, Cairns Australia, 2007.

[4]. V. Hariharan, P.S.S.Srinivasan, Vibration analysis of flexible coupling by considering unbalance, World applied sciences journal(8),2010.

[5]. K.M.AI-Hussian, I. Redmond, Dynamic Response of Two Rotors Connected by Rigid Mechanical Coupling with Parallel Misalignment, Journal of Sound and Vibration 249(3), (2002), pg. 483-498

[6]. G.N.D.S. Sudhakar, A.S. Sekhar - Identification of Unbalance in A Rotor Bearing Systemll, Journal of Sound and Vibration , (2011),330 2299-2313

[7]. J. Piotrowski ,Shaft Alignment Handbookll, 3rd edition M. Dekkor, Inc New York U.S.A, (2006)

[8]. Robert Randell,Vibration based condition monitoring , John Wiley publication USA, (2008)

[9]. http://www.vibrationschool.com/mans/SpecInter/SpecInter06.htm 